\title{
Netrin-4 Delays Colorectal Cancer Carcinomatosis by Inhibiting Tumor Angiogenesis
}

\author{
Clarisse Eveno, ${ }^{*}$ Dong Broqueres-You, ${ }^{\star \dagger}$ \\ Jean-Guillaume Feron, ${ }^{\star \dagger}$ Aurore Rampanou, ${ }^{*}$ \\ Annemilaï Tijeras-Raballand, ${ }^{* \dagger}$ Stanislas Ropert, ${ }^{* \dagger}$ \\ Laurence Leconte, ${ }^{\star \dagger}$ Bernard I. Levy, ${ }^{\star \dagger}$ \\ and Marc Pocard* \\ From INSERM U965 (Paris 7 University)* and the Vessels and \\ Blood Institute (IVS), ${ }^{\dagger}$ Hôpital Lariboisière, Paris, France
}

A close relationship between tumor angiogenesis, growth, and carcinomatosis has been observed. Netrin-4 (NT-4) has been shown to regulate angiogenic responses. We aimed to examine the effects of NT-4 on colon tumor angiogenesis, growth, and carcinomatosis. We showed that NT-4 was expressed in human colon cancer cells (LS174). A 20-fold increase in NT-4 expression was stably induced by NT-4 pcDNA in LS174 cells. In vivo, a Matrigel angiogenesis assay showed that NT-4 overexpression altered vascular endothelial growth factor (VEGF)/basic fibroblast growth factor-induced angiogenesis. In nude mice with LS174 xenografts, NT-4 overexpression inhibited tumor angiogenesis and growth. In addition, these NT-4-involved inhibitory effects were associated with decreased tumor cell proliferation and increased tumor cell apoptosis. Using an orthotopic peritoneal carcinomatosis model, we demonstrated that NT-4 overexpression decreased colorectal cancer carcinomatosis. Moreover, carcinomatosis-related ascites formation was significantly decreased in mice transplanted with NT-4 LS174 cells versus control LS174 cells. The antiangiogenic activity of NT- 4 was probably mediated by binding to its receptor neogenin. Netrin- 4 had a direct effect on neither in vitro apoptosis and proliferation of cultured LS174 cells nor the VEGF-induced acute increase in vascular permeability in vivo. We propose that NT-4 overexpression decreases tumor growth and carcinomatosis, probably via an antiangiogenic effect, underlying the potential therapeutic interest in NT-4 in the treatment of colorectal cancer growth and carcinomatosis. (Am J Pathol 2011, 178:1861-1869; DOI: 10.1016/j.ajpath.2010.12.019)
Colorectal cancer (CRC) is the second leading cause of cancer death in North America and Western Europe, constituting approximately $10 \%$ of new colon cancer cases every year. ${ }^{1,2}$ Despite recent progress in the treatment of CRC, for $19 \%$ of patients diagnosed as having metastatic CRC, survival times are poor, with 5-year survival being just $10 \%{ }^{3}$ Indeed, the survival rate is negatively related to the presence of carcinomatosis, a specific metastatic condition leading to significant morbidity and mortality. The development of new therapeutic strategies for $\mathrm{CRC}$ carcinomatosis is very much required, with the aim of delaying metastatic cancer progression and increasing survival rates.

Aberrant angiogenesis, the formation of new blood vessels from preexisting blood vessels and vascular endothelial cells (EC), is important in cancer progression. ${ }^{4}$ In neoplasms, tumor-derived factors promote angiogenesis. ${ }^{5}$ The neovascularization expands the vascular bed, causing tumor growth and a continuity with the systemic circulation; these changes provide a critical growth advantage for tumor progression and metastasis, ${ }^{6,7}$ suggesting that targeting the tumor's dependence on angiogenesis could, effectively, slow metastatic progression. Recently, several new drugs targeting specific molecules involved in angiogenesis have clinically validated this conjecture. $^{8}$ Because elevated vascular endothelial growth factor (VEGF) levels have been documented in many cancers, and VEGF overexpression correlates with an increased risk of metastatic disease and overall poor prognosis in different cancers, researchers have focused on the inhibition of VEGF/VEGF receptor pathways. ${ }^{9-13}$ Indeed, VEGF inhibitors are in broad use for the treatment of metastatic renal cell carcinoma, gastrointestinal stromal tumors, and hepatocellular carcinoma, and are being developed for the treatment of many other cancers, including $\mathrm{CRC}$, non-small cell lung cancer, and pancreatic cancer. ${ }^{8}$ However, experience with biological antiangiogenic agents, such as bevacizumab, indicates that disruption of VEGF

\footnotetext{
This study was supported in part by Association pour la Recherche sur le Cancer of France.

Accepted for publication December 17, 2010.

Address reprint requests to Dong Broqueres-You, M.D., Ph.D., Vessels and Blood Institute, INSERM U965 (Paris 7 University), Hôpital Lariboisière, 8 rue Guy Patin, 75010 Paris, France. E-mail: youdong777@hotmail.com.
} 
function often leads to severe secondary effects, such as delayed wound healing, renal damage, and subsequent hypertension. ${ }^{14}$ In addition, recent studies showed a resistance of tumor vessels to anti-VEGF therapy, ${ }^{15-17}$ highlighting the importance of developing other potential antiangiogenic strategies for cancer treatment.

Neuronal and vascular development requires guidance to establish a precise branching pattern of these systems in the vertebrate body. Several molecules implicated in axon navigation have also been shown to regulate vessel sprouting. ${ }^{18,19}$ Among these guidance cues, netrins constitute a family of diffusible molecules with a regulatory role in axon pathfinding and in other developmental processes, including vascular development. ${ }^{18}$ The netrin system comprises at least five ligands (netrins 1, 2, 4, G1a, and G1b) and seven receptors (neogenin, DCC, Unc5A, Unc5B, Unc5C, Unc5D, and A2b). ${ }^{20,21}$ Although the significance of the netrin-dependent pathway in tumor angiogenesis is not totally understood, emerging evidence indicates that it plays an important role in the development of different cancers in animal models. ${ }^{20}$ It might, therefore, be a potential therapeutic target for cancer treatment. A previous study showed that netrin-4 (NT-4) overexpression delayed tumor angiogenesis through binding to neogenin and recruitment of Unc5B in an s.c. xenograft model. ${ }^{22}$ In addition, a recent study showed that NT-4 regulated angiogenic responses and inhibited the growth of CRC cells. ${ }^{23}$

The aim of this study was to examine the effects of NT-4 overexpression on tumor angiogenesis and growth in vitro in human colon cancer cells (LS174) and in vivo in a mouse model of orthotopic peritoneal CRC carcinomatosis. We identified NT-4 as a protein expressed in vitro in LS174 human colon cancer cells. We demonstrated an inhibitory role of NT-4 in tumor progression by an effect on tumor angiogenesis but not by directly altering tumor cell proliferation and apoptosis. Finally, we obtained evidence that NT-4 exerts antiangiogenic activity, probably by binding to neogenin, and decreases CRC carcinomatosis and ascites formation.

\section{Materials and Methods}

\section{Cell Culture and Cell Transfection}

LS174 human colon cancer cells obtained from the American Type Culture Collection were maintained in Dulbecco's modified Eagle's medium supplemented with 10\% heat-inactivated fetal bovine serum/penicillin $(50 \mathrm{U} / \mathrm{mL}) /$ streptomycin (50 $\mu \mathrm{g} / \mathrm{mL})$ (Gibco BRL Life Technologies Inc, Grand Island, NY) in a humidified atmosphere of 95\% air and $5 \% \mathrm{CO}_{2}$ at $37^{\circ} \mathrm{C}$.

\section{Acquisition of cDNA for NT-4}

Total RNA was extracted from cultures of human umbilical artery endothelial cells, and reverse transcription was performed using the AMV Kit (Roche Applied Science, Indianapolis, IN). Two sets of primers (1-1039 and 9141884) were used to amplify the sequence with Taq DNA polymerase (Roche Applied Science). Products from the two PCR reactions were mixed 1:1 and were used as the template to obtain NT-4 cDNA. The construct was subcloned into a pcDNA3.1(-)/His-myc C vector (Invitrogen, Carlsbad, CA). The vector containing NT-4 was transfected into LS174 cells using Lipofectin (Invitrogen) according to the manufacturer's protocol. Cells were cultured in a selective medium ( $10 \%$ fetal bovine serum-Dulbecco's modified Eagle's medium) containing 200, 300, 400, or 500 $\mu \mathrm{g} / \mathrm{mL}$ of G418 (Geneticin; Gibco BRL Life Technologies Inc). Cell clones were subsequently screened by means of enzyme-linked immunosorbent assay to detect an increase in NT-4 expression relative to that in the pcDNA-transfected cells collected after 3 days in conditioned medium (data not shown). For in vivo experiments, LS174 cells transfected with NT-4 were harvested. Cells were washed in 10\% fetal bovine serum-Dulbecco's modified Eagle's medium, counted, and suspended in Dulbecco's modified Eagle's medium for injection.

\section{Matrigel Angiogenesis Assay}

A modified in vivo assay of neovessel growth, as described by Passaniti et al, ${ }^{24}$ was performed. Under anesthesia, $300 \mu \mathrm{L}$ of Matrigel (BD Biosciences, Franklin Lakes, NJ), $12.5 \mathrm{mg} / \mathrm{mL}$ mixed with PBS, or $6 \mu \mathrm{g} / \mathrm{mL}$ of NT-4 was s.c. injected into female athymic nude mice (5 weeks old) along the dorsal midline in the presence or absence of $300 \mathrm{ng} / \mathrm{mL}$ of VEGF-A and $300 \mathrm{ng} / \mathrm{mL}$ of basic fibroblast growth factor (bFGF) (R\&D Systems, Minneapolis, MN). After 7 days, the Matrigel plugs were harvested from underneath the skin. The plugs were homogenized in $1 \mathrm{~mL}$ of HEPES (20 mmol/L, $\mathrm{pH}$ 7.4) on ice and were cleared by means of centrifugation at 10,000 rpm for 6 minutes at $4^{\circ} \mathrm{C}$. The supernatant was collected and used to measure hemoglobin content using Drabkin's reagent along with a hemoglobin standard according to the manufacturer's protocol (Sigma-Aldrich, St. Louis, MO).

\section{Animal Models of Tumor Xenograft and Peritoneal Carcinomatosis}

All the experimental protocols met all the standards required by the European community guidelines for the care and use of laboratory animals. Five-week-old female athymic nude mice (Charles River Laboratories International Inc., Wilmington, MA) were acclimatized for 1 to 2 weeks before tumor transplantation (8 to 10 animals per experimental group).

For LS174 s.c. transplantation, animals were anesthetized with $2 \mathrm{mg} / \mathrm{mL}$ of xylazine and $20 \mathrm{mg} / \mathrm{mL}$ of ketamine (Virbac, Carros, France). Under sterile conditions, either NT-4-transfected LS174 cells (NT-4 LS174 cells) or control LS174 cells (CT LS174 cells; $1 \times 10^{6}$ cells) were injected s.c. The tumor volume was recorded using a caliper twice a week and was calculated using the following formula: [length $\left.(\mathrm{mm}) \times[\text { width }(\mathrm{mm})]^{2} \times \mathrm{p}\right] / 6$. Animals were sacrificed 30 days after LS174 transplantation.

For the peritoneal carcinomatosis orthotopic model, the animals were anesthetized. Under sterile conditions, either NT-4 or CT LS174 cells $\left(1 \times 10^{6}\right.$ cells $)$ 
were injected into the peritoneal cavity. Beginning 7 days after tumor transplantation, the mice received intravenous treatments consisting of bevacizumab (5 $\mathrm{mg} / \mathrm{kg}$ ) or saline twice per week for 3 weeks. The animals were monitored daily and were sacrificed at 30 days. Ascitic fluid was collected by performing a lower midline incision, and ascites volume was measured. The incision was then extended to allow photography of the peritoneal cavity and determination of the extent of peritoneal carcinomatosis using a modified Peritoneal Cancer Index (PCI). ${ }^{25}$ The $\mathrm{PCl}$ (range, 1 to 39) allows assessment of the distribution of cancer in the abdomen and pelvis and is calculated by summing lesion size scores (0 to 3 ) in the abdominopelvic regions (0 to 13). The $\mathrm{PCl}$ was adapted to tumor sizes in mice with the following lesion size scores: tumor smaller than $2.0 \mathrm{~mm}$ (lesion size 1), 2.1 to $5.0 \mathrm{~mm}$ (lesion size 2), and greater than $5.0 \mathrm{~mm}$ or confluence (lesion size 3), as previously described. ${ }^{26}$ Tumor tissue was then harvested and frozen for subsequent immunohistochemical analysis.

\section{Immunohistologic Analysis}

For the evaluation of CD31 and Ki-67 staining, each frozen tumor sample was sectioned at $5-\mu \mathrm{m}$ thickness using a cryostat (Leica Microsystems GmbH, Wetzlar, Germany). The sections were incubated with anti-CD31 antibody, anti-desmin antibody, anti-Ki-67 antibody, and DAPI (BD Biosciences) for 1 hour at room temperature and then with the second antibody. Images were acquired using a fluorescence microscope equipped with appropriate filters (Observer.Z1; Carl Zeiss Inc). HistoLab software (version 7.0; Microvision Instruments, Evry, France) was used to quantify the results. The results are expressed as the ratio of CD31 staining surface to desmin staining surface and the ratio of Ki-67 staining surface to DAPI staining surface.

\section{Cell Proliferation and Apoptosis Assays}

To evaluate in vitro tumor cell proliferation and apoptosis, CT and NT-4 LS174 cells were maintained under normal growth conditions, described previously herein. One hundred thousand cells were seeded in triplicate into each well of a 6-well flat-bottom plate. The cells were allowed to attach and grow overnight. Tumor cell proliferation was assessed at days $0,3,4,5$, and 7 by counting the cells using a Neubauer counting chamber. Using a terminal deoxynucleotidyl transferase-mediated dUTP nick-end labeling (TUNEL) kit (Roche Diagnostics), the apoptosis of LS174 cells was determined in frozen sections of each tumor xenograft sample and in cultured CT LS174 and NT-4 LS174 cells at day 7 . The ratio of surface occupied by TUNEL staining to total surface examined for the cross-sections and the percentage of TUNELstained cells to DAPI-stained cells for the cultured LS174 in vitro were determined using a customized application (Histolab).

\section{Western Blot Analysis}

At day 7 of cell culture, NT-4 LS174, CT LS174, and human umbilical vein endothelial cells (HUVEC) were homogenized in radioimmunoprecipitation assay lysis buffer [50 mmol/L Tris- $\mathrm{HCl}, 150 \mathrm{mmol} / \mathrm{L} \mathrm{NaCl}, 1 \mathrm{mmol} / \mathrm{L}$ EDTA, 1\% Triton X-100 (Roche Diagnostics $\mathrm{GmbH}$, Mannheim, Germany), 0.1\% SDS, 1\% deoxycholate, $\mathrm{pH}$ 7.4] containing protease inhibitors (Boehringer Mannheim Corp., New York, NY). Proteins were separated in $4 \%$ to $12 \%$ gradient SDS-polyacrylamide gel electrophoresis precast gels and were blotted onto a nitrocellulose sheet (Amersham Biosciences, Piscataway, NJ). Antibodies directed against NT-4, actin, and neogenin (R\&D Systems) were used at a dilution of 1:500, 1:400, and $1: 500$, respectively. Specific protein was detected by chemiluminescent reaction (Amersham Biosciences).

\section{Acute Vascular Permeability Assay}

Vascular endothelial growth factor has been reported to exert angiogenic effects by augmenting vascular permeability. ${ }^{9}$ To investigate the acute effect of NT-4 on vascular permeability, an intradermal Miles assay was performed. ${ }^{27}$ Briefly, the guinea pigs $(n=6$; Charles River Laboratories International Inc.) were anesthetized. A total of $500 \mu \mathrm{L}$ of $0.5 \%$ Evans blue (Sigma-Aldrich) was injected into animals via the jugular vein. After 30 minutes, the guinea pigs were given multiple 100- $\mu \mathrm{L}$ intradermal injections on the back. PBS, VEGF-A (5, 10, and $100 \mathrm{ng} / \mathrm{mL})$, VEGF-A (5, 10, and $100 \mathrm{ng} / \mathrm{mL})+\mathrm{NT}-4(10 \mu \mathrm{g} / \mathrm{mL})$, or VEGF-A (5, 10 , and $100 \mathrm{ng} / \mathrm{mL})+$ bevacizumab $(5 \mu \mathrm{g} / \mathrm{mL})$ was then injected intradermally. Twenty minutes after VEGF treatment, the animal was sacrificed and the skin was excised. Evans blue dye in the excised site of intradermal injection was photographed. For quantification of extravasated dye, skin samples were incubated for 48 hours in formamide at $4^{\circ} \mathrm{C}$, and the dye quantity was measured spectrophotometrically at $620 \mathrm{~nm}$.

\section{EC Tube Formation Assay}

HUVEC were cultured in endothelial basal medium (Clonetics, Lonza Walkersville Inc, Walkersville, MD) supplemented with $10 \%$ fetal bovine serum. The HUVEC cells $\left(6 \times 10^{4}\right)$ were then seeded in duplicate in a 24-well plate precoated with $10 \mathrm{mg} / \mathrm{mL}$ of Matrigel. Capillary-like tube formation was induced by the addition of $50 \mathrm{ng} / \mathrm{mL}$ of VEGF for 2 hours. Then, CT and NT-4 LS174 cells were added into wells in either the presence or absence of 10 $\mu \mathrm{g} / \mathrm{mL}$ of neutralizing antibody against neogenin. Eighteen hours later, the media was removed and the cells were washed and stained with $4 \mu \mathrm{g} / \mathrm{mL}$ of calcein (Invitrogen) in a PBS solution at $37^{\circ} \mathrm{C}$. Endothelial cell tube formation was quantified by determining the number of new EC tubes. 
A

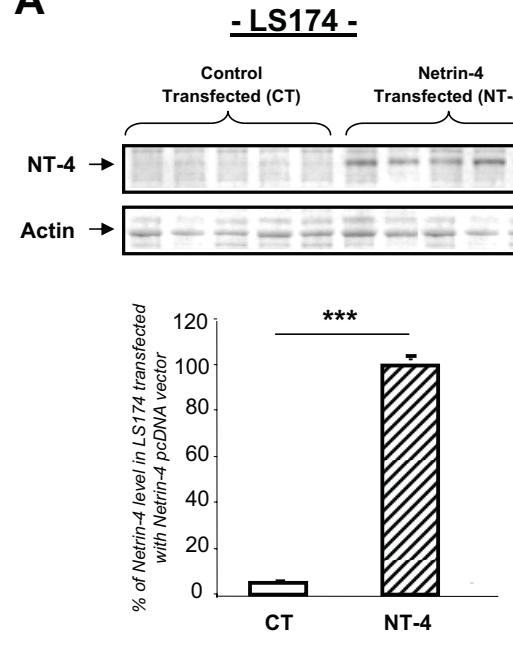

B

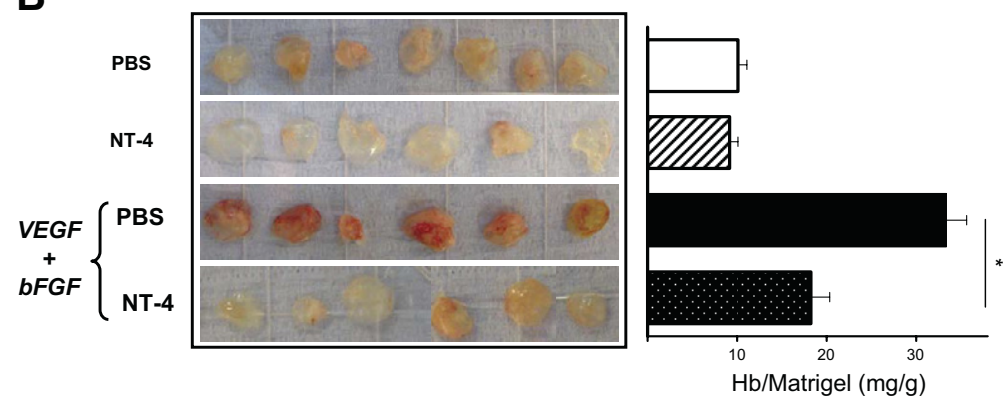

Figure 1. Expression of NT-4 and the effect of NT-4 on VEGF/bFGF-induced nontumoral angiogenesis. A: NT-4 is expressed in LS174 human CRC cells, and its expression is stably increased by NT-4 pcDNA vector transfection. B: NT-4 does not modify angiogenesis in the absence-of-VEGF/ bFGF control condition. However, NT-4 inhibits VEGF/bFGF-induced angiogenesis in Matrigel plugs. Hb, hemoglobin. Error bars represent SEM. ${ }^{*} P<0.05$; ${ }^{* * *} P<0.001$

\section{Statistical Analysis}

The results are expressed as mean \pm SEM. The unpaired t-test was used to compare two different experimental groups. The comparisons between more than two different experimental groups were performed using 1-way analysis of variance followed by a Bonferroni posttest. A $P<0.05$ was considered significant.

\section{Results}

\section{NT-4 Is Expressed in Human Colon Cancer Cells}

The protein expression of NT-4 was determined by means of Western blot analysis (Figure 1A). After transfection with the NT-4 pcDNA vector, LS174 human colon cancer cells exhibited a 20-fold increase in NT-4 level compared with CT LS174 cells, with this increase being reproducible and stable $(P<0.001$; Figure $1 \mathrm{~A})$.

\section{NT-4 Alters VEGF/Basic FGF-Induced Nontumoral Angiogenesis In Vivo}

We first investigated the role of NT-4 in angiogenesis induced by VEGF/bFGF, the key factors involved in physiologic and pathologic angiogenesis. Recombinant NT-4 protein did not modify the hemoglobin content in the absence-of-VEGF/bFGF control condition (mean \pm SEM: $10.1 \pm 1.0 \mathrm{mg} / \mathrm{g}$ and $9.2 \pm 0.9 \mathrm{mg} / \mathrm{g}$ for the PBS and NT-4 groups, respectively; Figure 1B). Seven days after VEGF-A and FGF-2 treatment, increased hemoglobin content was observed using an angiogenesis assay in Matrigel, confirming that VEGF-A and FGF-2 promote angiogenesis. Recombinant NT-4 protein significantly inhibited this angiogenesis induced by VEGF and FGF (mean \pm SEM: $33.4 \pm 2.3 \mathrm{mg} / \mathrm{g}$ and $10.1 \pm$ $1.0 \mathrm{mg} / \mathrm{g}$ for the VEGF/bFGF + PBS and VEGF/bFGF + NT-4 groups, respectively; $P<0.5$; Figure $1 \mathrm{~B}$ ).
NT-4 Inhibits Colorectal Tumor Growth, Impairs Tumor Angiogenesis, and Affects Tumor Cell Proliferation and Apoptosis in Human Colon Cancer Cell Xenograft

To examine whether NT-4 could decrease colon tumor growth and angiogenesis, CT and NT-4 LS174 human colon cancer cells were s.c. injected into nude mice. In animals with an NT-4 LS174 xenograft, the tumor volume was significantly smaller than that of animals receiving CT LS174 cells (Figure 2A). The VEGF inhibitor bevacizumab had no significant effect on tumor growth. In addition, bevacizumab did not further decrease the tumor volume in animals transplanted with NT-4 LS174 cells (Figure 2A).

Decreased tumor progression was related to a reduction in the ratio of CD31 to desmin staining surface in animals in the NT-4 group, suggesting that NT-4 exerts an inhibitory effect on tumor angiogenesis (mean \pm SEM: $0.73 \pm 0.05$ and $0.47 \pm 0.06$ for the CT and NT-4 groups, respectively; $P<0.05$; Figure $2 \mathrm{~B}$ ).

Moreover, NT-4 overexpression dramatically decreased the ratio of $\mathrm{Ki}-67$ to DAPI staining surface in the tumor, indicating that NT-4 impairs tumor proliferation in vivo (mean \pm SEM: $1 \pm 0.14$ and $0.48 \pm 0.07$ for the CT and NT-4 groups, respectively; $P<0.01$; Figure $2 \mathrm{C}$ ). Tumor cell apoptosis detected by means of TUNEL staining was greatly increased in the NT-4 group compared with the CT group (mean \pm SEM: $0.12 \pm 0.02$ and $0.32 \pm 0.10$ for the CT and NT-4 groups, respectively; $P=0.05$; Figure 2D).

\section{NT-4 Has No Direct Effect on Tumor Cell Proliferation and Apoptosis in Vitro}

To ascertain whether NT-4 directly affects the proliferation and apoptosis of colon cancer cells, we investigated the effect of NT-4 overexpression on proliferation and apoptosis of LS174 cells in vitro. There was no difference in cell numbers between the CT and NT-4 LS174 dishes during the 7-day cell culture period, indicating that NT-4 did not directly alter the proliferation of human colon cancer cells 
A

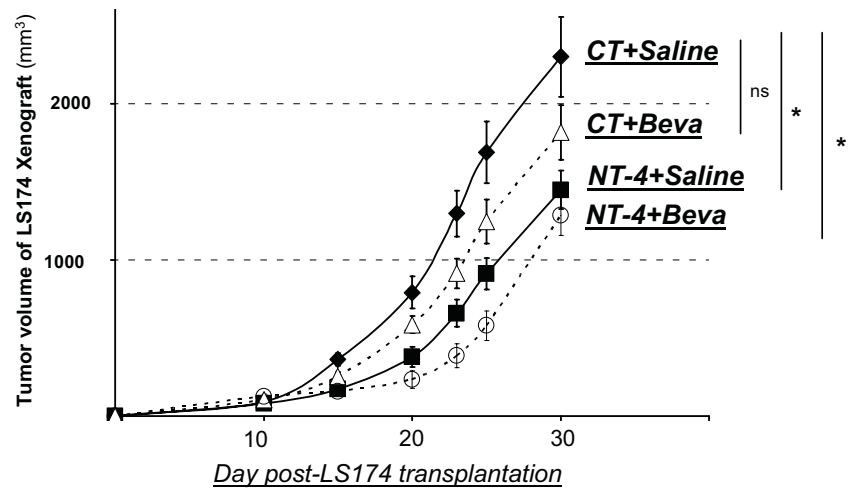

B

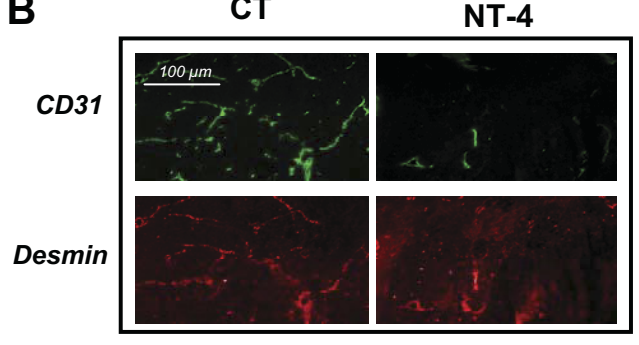

C

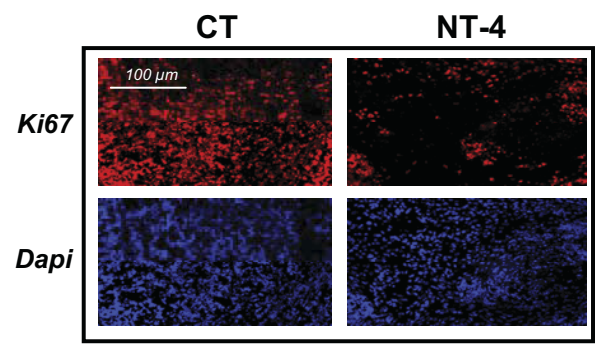

D

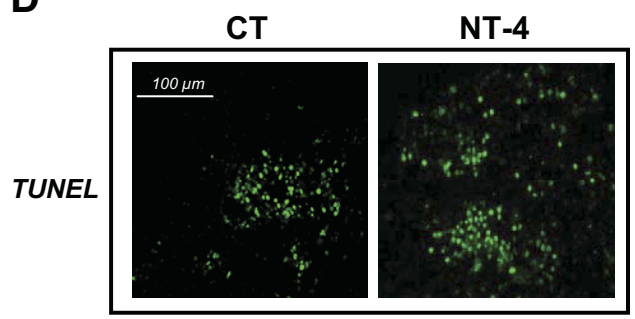

Tumor Angiogenesis (CD31/Desmin surface)

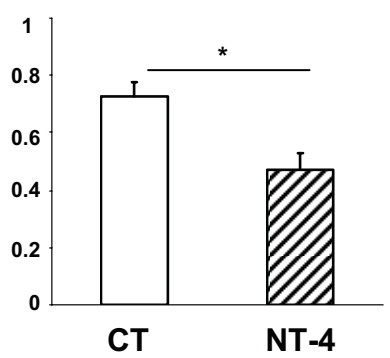

Tumor Proliferation (Ki67/Dapi surface)

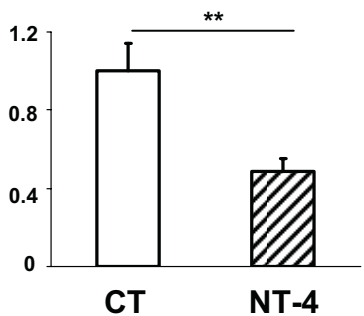

Tumor Apoptosis (TUNEL/Total examined surface)

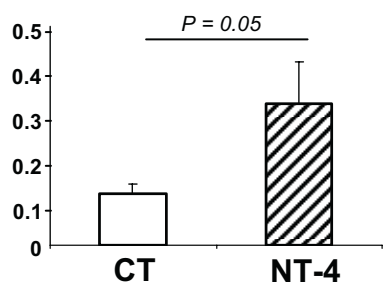

Figure 2. Effects of NT-4 and bevacizumab (Beva) on colorectal tumor growth, tumor angiogenesis, tumor cell proliferation, and apoptosis in nude mice with an s.c. LS174 cell xenograft. A: NT-4 overexpression in LS174 cells delays tumor growth, whereas bevacizumab has no significant inhibitory effect. B: Vascular density (CD31/desmin staining surface) is significantly less pronounced in tumors transplanted with NT-4-overexpressing LS174 cells compared with CT LS174 cells. C: NT-4 overexpression in LS174 cells is related to a significant decrease in tumor cell proliferation (Ki-67/DAPI staining surface) in vivo. D: NT-4 overexpression in LS174 cells significantly increases tumor apoptosis in vivo. ns, not significant. Error bars represent SEM. ${ }^{*} P<0.05 ;{ }^{* *} P<0.01$.

(Figure 3A). Moreover, NT-4 overexpression did not significantly modify the tumor cell apoptotic process in vitro (Figure 3B).

\section{NT-4 Decreases CRC Carcinomatosis and lts Related Ascites Formation}

We next examined the effects of NT-4 overexpression, compared with bevacizumab, on CRC carcinomatosis using a modified $\mathrm{PCl}$ assessment. Day 30 after cancer cell transplantation, the $\mathrm{PCl}$ was significantly decreased in animals receiving NT-4 LS174 cells compared with CT LS174 cells, suggesting that NT-4 affects the distribution of transplanted cancer in the abdomen and pelvis (mean \pm SEM: $18.6 \pm 3.7$ and $7.8 \pm 1.6$ for the CT and NT-4 groups, respectively; $P<0.05$; Figure $4, \mathrm{~A}$ and $\mathrm{C}$ ). The VEGF inhibitor bevacizumab had no effect on the PCl. Moreover, bevacizumab could not further enhance the 


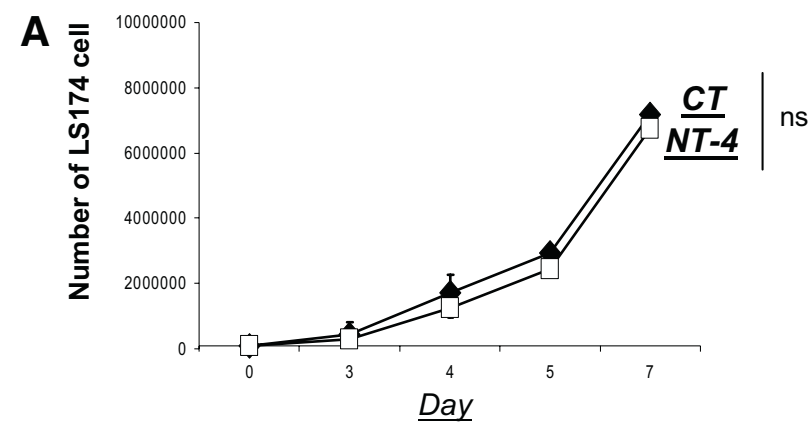

B
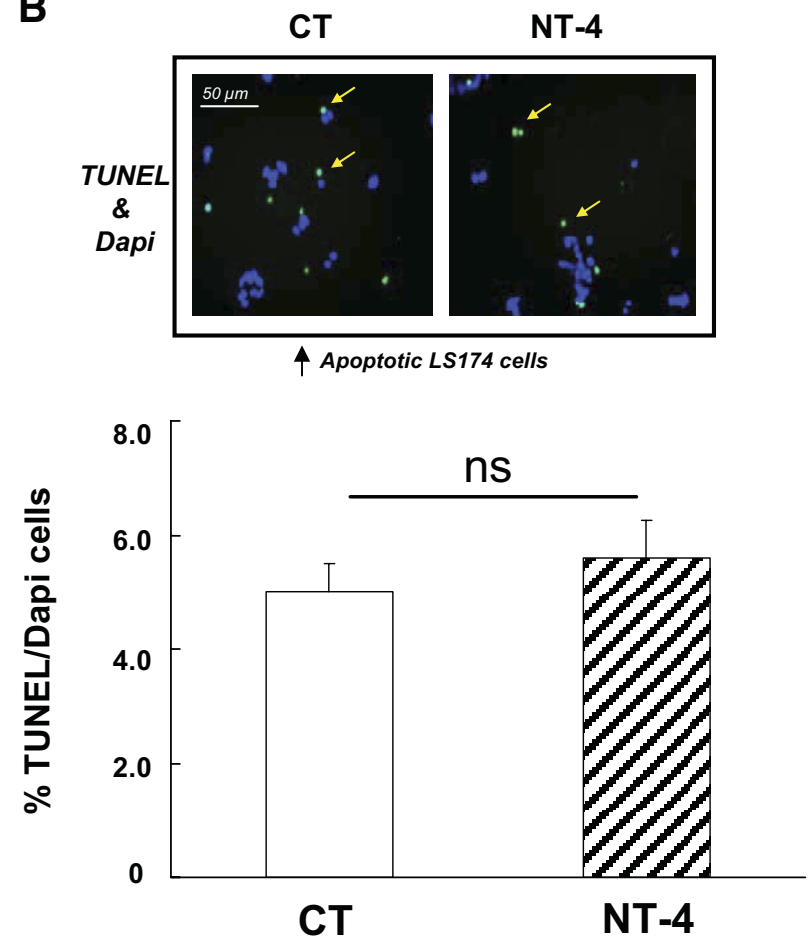

Figure 3. In vitro effects of NT-4 overexpression on colorectal tumor cell proliferation and apoptosis. A: NT-4-overexpressing LS174 cells exhibit a similar proliferation capability as CT LS174 cells. B: NT-4-overexpression has no significant effect on tumor apoptosis. ns, not significant; TUNEL, terminal deoxynucleotidyl transferase-mediated dUTP nick-end labeling. Error bars represent SEM.

inhibitory effect of NT-4 on CRC carcinomatosis (Figure 4A).

Colorectal cancer carcinomatosis is often related to ascites formation. Both NT-4 and bevacizumab were capable of significantly decreasing the ascites volume in nude mice with LS174 transplantation (mean \pm SEM: $0.94 \pm 0.23,0.16 \pm 0.06,0.13 \pm 0.06$, and $0.13 \pm 0.07$ $\mathrm{mL}$ for the CT, NT-4, bevacizumab, and NT-4 + bevacizumab groups, respectively; $P<0.05$; Figure 4B). However, bevacizumab did not further decrease ascites formation in animals injected with NT-4 LS174 cells.

Qualitative photography showed fewer blood vessels in the parietal peritoneum (Figure 4C) and abdominal cavity (Figure 4D) in the NT-4 group compared with the CT group, suggesting that NT-4 has an inhibitory effect on tumor angiogenesis of CRC carcinomatosis.

\section{NT-4 Has No Direct Effect on a VEGF-Induced Acute Increase in Vascular Permeability}

Then, we investigated the acute effect of NT-4 on vascular permeability using an intradermal Miles in vivo permeability assay in the guinea pig, with or without the presence of VEGF. As originally described by Miles and Miles, ${ }^{27}$ rapid leakage of Evans blue dye was observed in the skin of animals in the presence of VEGF in a dosedependent manner (Figure 5). Recombinant NT-4 protein did not modify the leakage of Evans Blue in the presence of VEGF, suggesting that NT-4 has no direct effect on a VEGF-induced acute increase in vascular permeability. However, bevacizumab totally blocked the VEGF-induced acute increase in vascular permeability (Figure 5).

\section{The Receptor Neogenin Mediates the Antiangiogenic Activity of NT-4}

To investigate the involvement of the receptor neogenin in NT-4's antiangiogenic activity, we used a Matrigel EC tube formation assay. First, the protein expression of neogenin was evidenced by means of Western blot analysis in HUVEC (Figure 6A). In the presence of VEGF, HUVEC were capable of forming new vascular tubes. Co-culture of HUVEC with LS174 cells significantly increased the number of new EC tubes (mean \pm SEM: $22 \pm 4,127 \pm 7$, and $328 \pm$ $30 / \mathrm{mm}^{2}$ for the PBS, VEGF, and VEGF + CT groups, respectively; $P<0.001$; Figure $6, \mathrm{~B}$ and $\mathrm{C}$ ), indicating that tumor cells facilitate EC angiogenesis. However, after a short-term (18-hour) cell culture, there was no difference in EC tube formation between the VEGF + CT and VEGF + NT-4 groups (mean \pm SEM: $293 \pm 19 / \mathrm{mm}^{2}$ for the VEGF + NT-4 group).

In the presence of neutralizing antibody directed against neogenin, the mean \pm SEM number of new vascular tubes was dramatically enhanced compared with the VEGF + CT and VEGF + NT-4 groups $\left(749 \pm 41 / \mathrm{mm}^{2}\right.$ for the VEGF + NT-4 + antineogenin group, $P<0.001$; Figure $6, \mathrm{~B}$ and $\mathrm{C}$ ), suggesting that neogenin mediates the antiangiogenic activity of LS174 cell-secreted NT-4.

\section{Discussion}

Interference with the activation of growth factor receptors and/or with intracellular growth factor-activated signal transduction pathways represents a promising strategy for the development of novel and selective anticancer therapies. With the clinical validation of several new drugs specifically targeting molecules involved in angiogenesis, antiangiogenic therapy is in broad use for the treatment of cancers. Indeed, more than 40 VEGF inhibitors are currently in development. ${ }^{8}$ The present study showed that bevacizumab (a humanized monoclonal antibody targeting the VEGF/VEGF receptor pathway) significantly decreases carcinomatosis-related ascites formation but alone is unable to cause a reduction in tumor growth and tumor carcinomatosis.

Recent studies have indicated that human cancers can acquire resistance after anti-VEGF treatments, ag- 


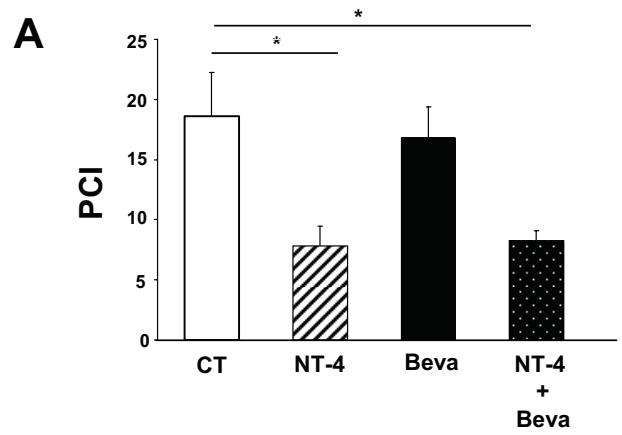

B
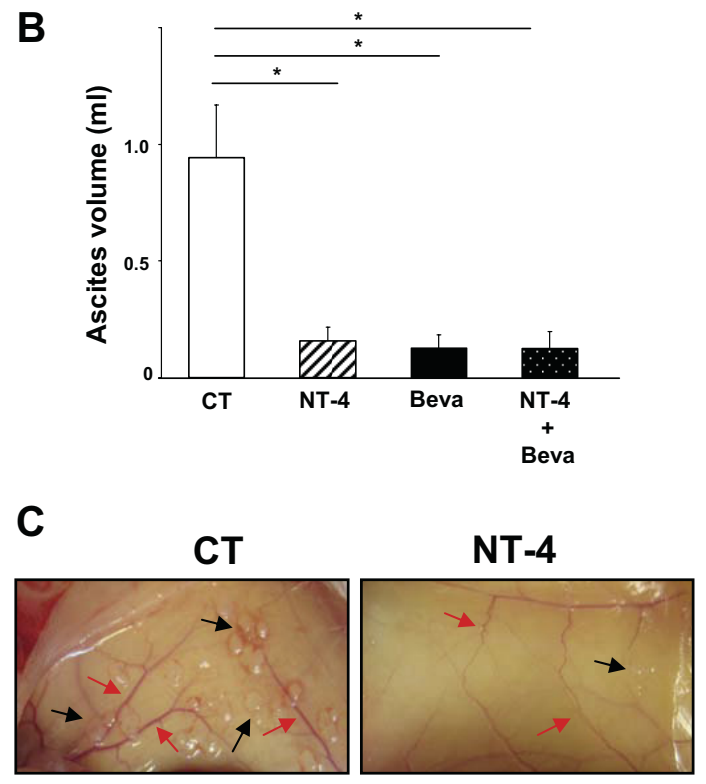

D
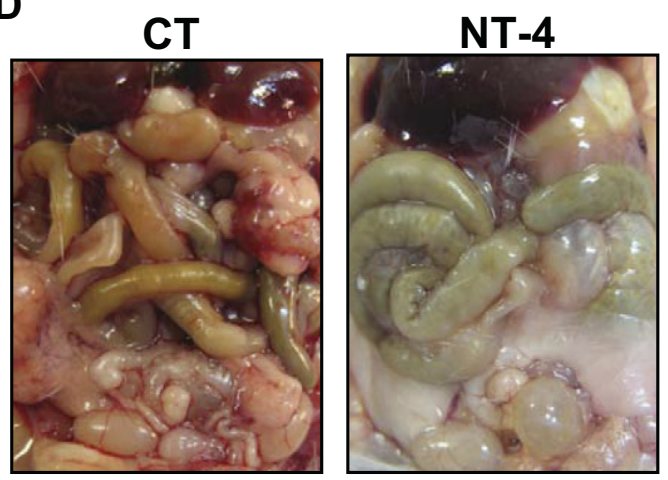

Figure 4. Effects of NT-4 on tumor carcinomatosis, ascites formation, and tumor angiogenesis in an orthotopic peritoneal carcinomatosis model in athymic mice. A: Overexpression of NT-4, but not bevacizumab (Beva) decreases the distribution of transplanted cancer in the abdomen and pelvis using modified PCI assessment. B: Both NT-4 overexpression in LS174 cells and Beva inhibit carcinomatosis-related ascites formation. C: Photography demonstrates that NT-4 overexpression in LS174 decreases tumor carcinomatosis and blood vessels in the parietal peritoneum. Black arrows indicate LS174 metastasis, red arrows indicate blood vessels. D: Photography shows fewer blood vessels in the abdominal cavity in the NT-4 group compared with the CT group, suggesting that NT-4 has an inhibitory effect on tumor angiogenesis. Error bars represent SEM. *P $P 0.05$.

gravating the pathologic situation. ${ }^{15-17}$ In addition, the disruption in VEGF function often leads to severe secondary effects, such as delayed wound healing, renal damage, and subsequent hypertension. ${ }^{14}$ As potential tar-
A

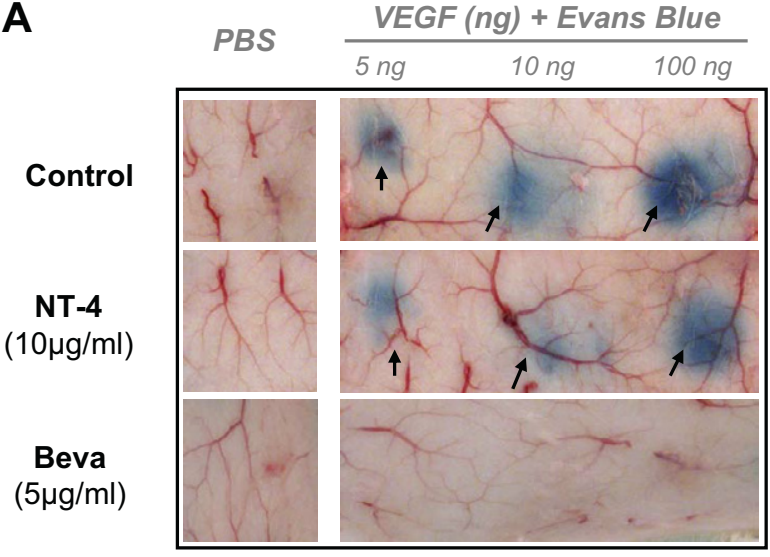

B

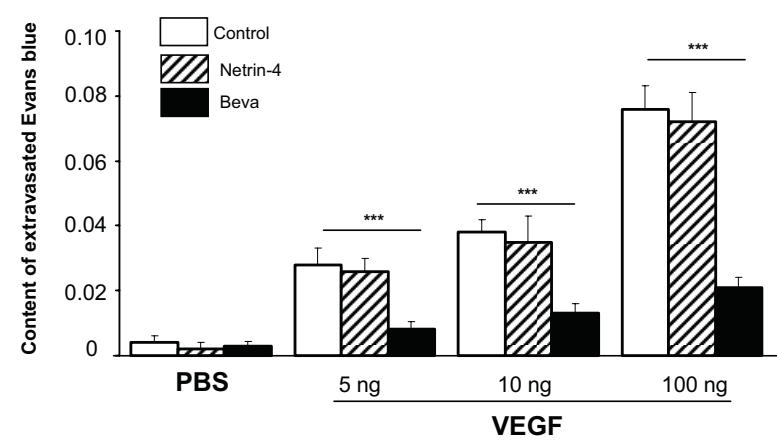

Figure 5. Acute effect of NT-4 on VEGF-induced augmentation of vascular permeability in guinea pig using Evans blue. A: Representative photos. Arrows indicate leakage of Evans blue in the skin. B: NT-4 has no effect on VEGFinduced acute augmentation of vascular permeability. In contrast, bevacizumab (Beva) efficiently inhibits the VEGF-induced acute increase in vascular permeability. Error bars represent SEM. ${ }^{* \cdots * * *} P<0.001$.

gets in cancer treatment, the netrin family is receiving more attention. Originally identified as axonal guidance molecules, netrins are laminin-like secreted proteins that have recently been shown to be involved in angiogenesis and blood vessel network formation. ${ }^{28}$ As observed in the nervous system, netrins also act as bifunctional modulators in angiogenesis. Indeed, both proangiogenic and antiangiogenic effects of netrin have been reported. ${ }^{29-31}$

Using Matrigel angiogenesis assay, we showed that NT-4 inhibits VEGF/bFGF-induced, nontumoral angiogenesis. In many cancers, elevated VEGF levels have been documented..$^{9-13}$ It has also been suggested that VEGF may induce the expression of antiangiogenic factors, such as thrombospondin and NT-4. We showed that NT-4 is expressed in LS174 human colon cancer cells and that NT-4 overexpression can be stably induced in these cells. Therefore, NT-4 could be a negative feedback regulator of pathologic angiogenesis, such as CRCrelated tumor angiogenesis. In fact, the present study demonstrated that NT-4 overexpression significantly decreases tumor growth in an s.c. CRC xenograft model, in accordance with results obtained by other research groups. ${ }^{22,23}$ Although this inhibited tumor growth is associated with decreased tumor cell proliferation and enhanced tumor cell apoptosis in vivo, it seems that NT-4 does not exert a direct effect on tumor proliferation and 
A

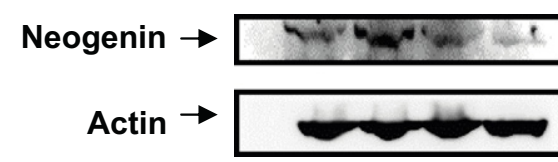

C

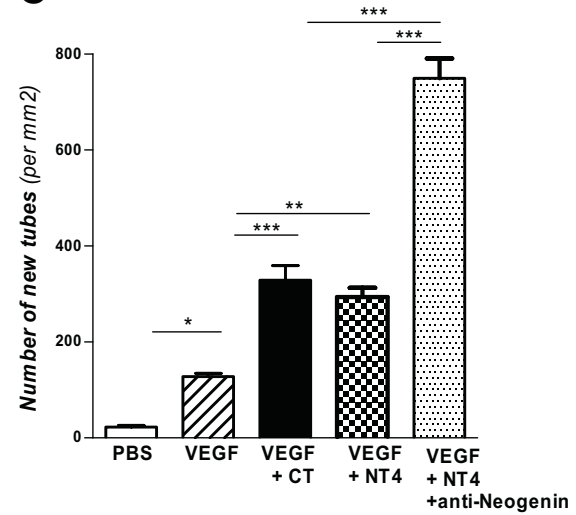

B

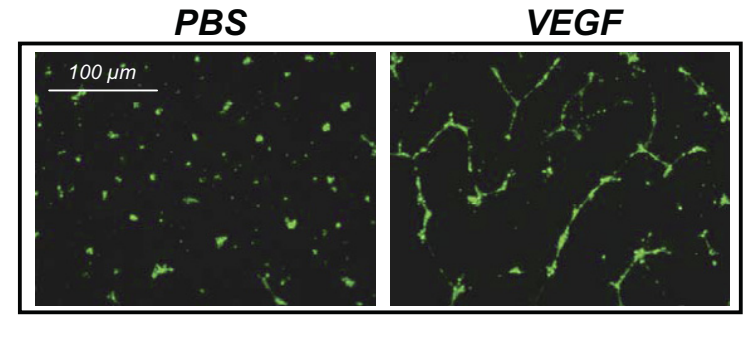

$V E G F+C T$ $V E G F+N T-4$
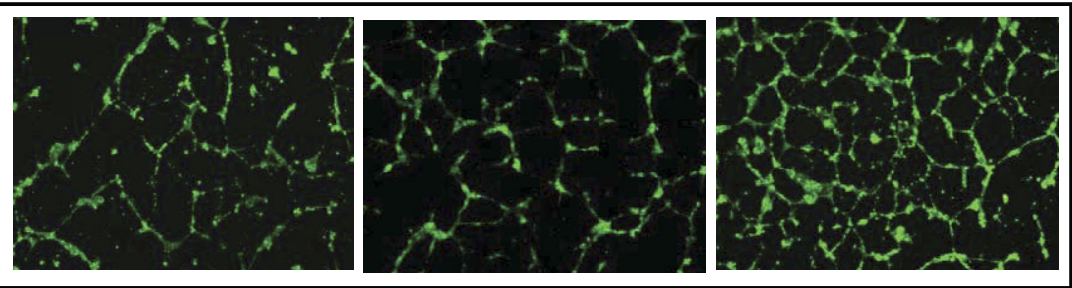

Figure 6. Involvement of neogenin in NT-4-related antiangiogenic activity in a Matrigel EC tube formation model. A: Neogenin is expressed in HUVEC. B: Representative images of Matrigel HUVEC tube formation from different experimental groups. C: In the presence of VEGF, the number of new EC tubes is enhanced by CT and NT-4 LS174 cells. Antibody directed against neogenin further increases EC tube formation. Error bars represent SEM. ${ }^{*} P<0.05 ;{ }^{* *} P<0.01$;

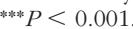

tumor apoptosis. Indeed, the present study showed that NT-4 overexpression has no direct in vitro effect on the proliferation of cultured LS174 cells. It has been reported that NT-4 inhibits in vitro proliferation of several tumor cell lines, such as pancreatic (Panc-1), liver (Hep3B), lung (SKLU1), and colon (HT29) tumor cell lines. ${ }^{23}$ The in vitro effect of NT-4 on tumor cell proliferation seems to be cell line-dependent. Our in vitro study showed that NT-4 overexpression has no direct effect on the apoptosis of cultured tumor cells, in accordance with a previous study. ${ }^{23}$ These results indicate that NT-4 does not exert an inhibitory effect on apoptosis of some tumor cell lines. A decrease in vascular EC is observed in tumor tissues after s.c. CRC xenograft, suggesting that NT-4 can decrease tumor proliferation, increase tumor apoptosis, and impair tumor growth by inhibiting tumor angiogenesis. Netrin-4 could exert this antiangiogenic effect by inhibiting the differentiation and migration of EC and stimulating the differentiation and migration of smooth muscle cells. ${ }^{22,23}$

In patients with $\mathrm{CRC}$, the survival rate is negatively related to the extent of carcinomatosis, an advanced tumor stage leading to significant morbidity and mortality. This study demonstrated that NT-4 overexpression decreases the distribution of transplanted CRC in the abdomen and pelvis, indicating that NT-4 could delay carcinomatosis processes and suggesting a potential therapeutic interest of NT-4 in the treatment of cancer and/or carcinomatosis. In contrast, bevacizumab alone does not modify colorectal tumor carcinomatosis.

Bevacizumab significantly decreases colon cancer carcinomatosis-related ascites. Indeed, capillary permeability, the overall capillary membrane surface area available for filtration, hydraulic pressure, and oncotic pressure are all involved in the pathogenesis of malignant ascites. Previous studies showed that VEGF can rapidly induce the augmentation of vascular permeability ${ }^{32,33}$; thus, bevacizumab could decrease ascites formation, probably by rapidly reducing vascular permeability. Using the acute vascular permeability assay in guinea pigs, the present results showed that bevacizumab significantly decreases VEGF-induced acute augmentation of vascular permeability.

We showed that NT-4 overexpression is also capable of reducing carcinomatosis-related ascites in a similar manner as bevacizumab. However, NT-4 protein cannot directly block VEGF-induced acute augmentation of vascular permeability, suggesting that NT-4 inhibits carcinomatosis-related ascites by different mechanisms than the action of bevacizumab. Interestingly, we demonstrated by means of photography that fewer blood vessels are observed in the parietal peritoneum and abdominal cavity in animals with intraperitoneal transplantation of NT-4 LS174 cells. Netrin-4 could exert this antiangiogenic effect by inhibiting the differentiation and migration of EC. ${ }^{22}$ These data suggest that NT-4 could inhibit ascites formation, at least in part, by decreasing the overall capillary membrane surface area available for filtration. In addition, it has been reported that NT-4 could stimulate the differentiation and migration of smooth muscle cells, ${ }^{22,23}$ suggesting that NT-4 could decrease the permeability of the tumor vascular network by chronically promoting vessel maturation. However, this hypothesis should be verified by further experiments.

The present study showed that co-culture with LS174 cells augments EC tube formation, indicating that tumor cells promote angiogenesis, in accordance with a previous study. ${ }^{34}$ It has been reported that NT-4 recombinant protein and NT-4 small interfering RNA could inhibit HUVEC tube 
formation. ${ }^{22,23}$ We did not detect a significant difference in EC tube formation between CT and NT-4 LS174 cells, probably owing to insufficient NT-4 protein secretion induced by NT-4 pcDNA vector transfection during a short-term (18hour) cell culture. The neutralizing antibody directed against neogenin, a receptor of NT-4, dramatically increases EC tube formation, strongly suggesting that neogenin mediates the antiangiogenic activity of NT-4. ${ }^{22,23}$ The activity of NT-4 is due to a baseline secretion of LS174 rather than to an overexpression of NT-4 induced by NT-4 pcDNA vector transfection.

In conclusion, NT-4 decreases tumor growth, delays carcinomatosis, and reduces ascites formation by inhibiting tumor angiogenesis. These findings highlight a therapeutic interest in NT-4 as a promising alternative to anti-VEGF treatments in the implementation of therapies against cancer.

\section{References}

1. Jemal A, Siegel R, Ward E, Hao Y, Xu J, Murray T, Thun MJ: Cancer statistics, 2008. CA Cancer J Clin 2008, 58:71-96

2. Lieberman D: Progress and challenges in colorectal cancer screening and surveillance. Gastroenterology 2010, 138:2115-2126

3. Ries LA, Wingo PA, Miller DS, Howe HL, Weir HK, Rosenberg HM, Vernon SW, Cronin K, Edwards BK: The annual report to the nation on the status of cancer, 1973-1997, with a special section on colorectal cancer. Cancer 2000, 88:2398-2424

4. Dvorak HF: Angiogenesis: update 2005. J Thromb Haemost 2005, 3:1835-1842

5. Ferrara N, Kerbel RS: Angiogenesis as a therapeutic target. Nature 2005, 438:967-974

6. Folkman J: Tumor angiogenesis: therapeutic implications. $\mathrm{N}$ Engl J Med 1971, 285:1182-1186

7. Zhong H, Bowen JP: Antiangiogenesis drug design: multiple pathways targeting tumor vasculature. Curr Med Chem 2006, 13:849-862

8. Ivy SP, Wick JY, Kaufman BM: An overview of small-molecule inhibitors of VEGFR signaling. Nat Rev Clin Oncol 2009, 6:569-579

9. Dvorak HF: Vascular permeability factor/vascular endothelial growth factor: a critical cytokine in tumor angiogenesis and a potential target for diagnosis and therapy. J Clin Oncol 2002, 20:4368-4380

10. Karkkainen MJ, Petrova TV: Vascular endothelial growth factor receptors in the regulation of angiogenesis and lymphangiogenesis. Oncogene 2000, 19:5598-5605

11. Nagy JA, Vasile E, Feng D, Sundberg C, Brown LF, Detmar MJ, Lawitts JA, Benjamin L, Tan X, Manseau EJ, Dvorak AM, Dvorak HF: Vascular permeability factor/vascular endothelial growth factor induces lymphangiogenesis as well as angiogenesis. J Exp Med 2002, 196:1497-1506

12. Schlessinger J, Ullrich A: Growth factor signaling by receptor tyrosine kinases. Neuron 1992, 9:383-391

13. Ullrich A, Schlessinger J: Signal transduction by receptors with tyrosine kinase activity. Cell 1990, 61:203-212

14. Eremina V, Jefferson JA, Kowalewska J, Hochster H, Haas M, Weisstuch J, Richardson C, Kopp JB, Kabir MG, Backx PH, Gerber HP, Ferrara N, Barisoni L, Alpers CE, Quaggin SE: VEGF inhibition and renal thrombotic microangiopathy. N Engl J Med 2008, 358:11291136

15. Helfrich I, Scheffrahn I, Bartling S, Weis J, von Felbert V, Middleton M, Kato M, Ergun S, Schadendorf D: Resistance to antiangiogenic therapy is directed by vascular phenotype, vessel stabilization, and maturation in malignant melanoma. J Exp Med 2010, 207:491-503

16. Huang D, Ding Y, Zhou M, Rini BI, Petillo D, Qian CN, Kahnoski R, Futreal PA, Furge KA, Teh BT: Interleukin-8 mediates resistance to antiangiogenic agent sunitinib in renal cell carcinoma. Cancer Res 2010, 70:1063-1071

17. Priceman SJ, Sung JL, Shaposhnik Z, Burton JB, Torres-Collado AX, Moughon DL, Johnson M, Lusis AJ, Cohen DA, Iruela-Arispe ML, Wu L: Targeting distinct tumor-infiltrating myeloid cells by inhibiting CSF-1 receptor: combating tumor evasion of antiangiogenic therapy. Blood 2010, 115:1461-1471

18. Freitas C, Larrivee B, Eichmann A: Netrins and UNC5 receptors in angiogenesis. Angiogenesis 2008, 11:23-29

19. Pasquale EB: Eph receptors and ephrins in cancer: bidirectional signalling and beyond. Nat Rev Cancer 2010, 10:165-180

20. Cirulli V, Yebra M: Netrins: beyond the brain. Nat Rev Mol Cell Biol 2007, 8:296-306

21. Dumartin L, Quemener C, Laklai H, Herbert J, Bicknell R, Bousquet C Pyronnet S, Castronovo V, Schilling MK, Bikfalvi A, Hagedorn M: Netrin-1 mediates early events in pancreatic adenocarcinoma progression, acting on tumor and endothelial cells. Gastroenterology 2010, 138:1595-1606.

22. Lejmi E, Leconte L, Pedron-Mazoyer S, Ropert S, Raoul W, Lavalette S, Bouras I, Feron JG, Maitre-Boube M, Assayag F, Feumi C, Alemany M, Jie TX, Merkulova T, Poupon MF, Ruchoux MM, Tobelem G, Sennlaub F, Plouet J: Netrin-4 inhibits angiogenesis via binding to neogenin and recruitment of Unc5B. Proc Natl Acad Sci U S A 2008, 105:12491-12496

23. Nacht M, St Martin TB, Byrne A, Klinger KW, Teicher BA, Madden SL, Jiang $Y$ : Netrin-4 regulates angiogenic responses and tumor cell growth. Exp Cell Res 2009, 315:784-794

24. Passaniti A, Taylor RM, Pili R, Guo Y, Long PV, Haney JA, Pauly RR, Grant DS, Martin GR: A simple, quantitative method for assessing angiogenesis and antiangiogenic agents using reconstituted basement membrane, heparin, and fibroblast growth factor. Lab Invest 1992, 67:519-528

25. Harmon RL, Sugarbaker PH: Prognostic indicators in peritoneal carcinomatosis from gastrointestinal cancer. Int Semin Surg Oncol 2005, 2:3

26. Otto J, Jansen PL, Lucas S, Schumpelick V, Jansen M: Reduction of peritoneal carcinomatosis by intraperitoneal administration of phospholipids in rats. BMC Cancer 2007, 7:104

27. Miles AA, Miles EM: Vascular reactions to histamine, histamine-liberator and leukotaxine in the skin of guinea-pigs. J Physiol 1952, 118:228-257

28. Eichmann A, Makinen T, Alitalo K: Neural guidance molecules regulate vascular remodeling and vessel navigation. Genes Dev 2005, 19:1013-1021

29. Larrivee B, Freitas C, Trombe M, Lv X, Delafarge B, Yuan L, Bouvree K, Breant C, Del TR, Brechot N, Germain S, Bono F, Dol F, Claes F, Fischer C, Autiero M, Thomas JL, Carmeliet P, Tessier-Lavigne M, Eichmann A: Activation of the UNC5B receptor by Netrin-1 inhibits sprouting angiogenesis. Genes Dev 2007, 21:2433-2447

30. Lu X, Le NF, Yuan L, Jiang Q, De Lafarge B, Sugiyama D, Breant C, Claes F, De SF, Thomas JL, Autiero M, Carmeliet P, Tessier-Lavigne M, Eichmann A: The netrin receptor UNC5B mediates guidance events controlling morphogenesis of the vascular system. Nature 2004, 432:179-186

31. Wilson BD, li M, Park KW, Suli A, Sorensen LK, Larrieu-Lahargue F, Urness LD, Suh W, Asai J, Kock GA, Thorne T, Silver M, Thomas KR, Chien CB, Losordo DW, Li DY: Netrins promote developmental and therapeutic angiogenesis. Science 2006, 313:640-644

32. Numnum TM, Rocconi RP, Whitworth J, Barnes MN: The use of bevacizumab to palliate symptomatic ascites in patients with refractory ovarian carcinoma. Gynecol Oncol 2006, 102:425-428

33. Wright JD, Hagemann A, Rader JS, Viviano D, Gibb RK, Norris L, Mutch DG, Powell MA: Bevacizumab combination therapy in recurrent, platinum-refractory, epithelial ovarian carcinoma: a retrospective analysis. Cancer 2006, 107:83-89

34. Agarwal A, Das K, Lerner N, Sathe S, Cicek M, Casey G, Sizemore N: The AKT/l $\kappa$ B kinase pathway promotes angiogenic/metastatic gene expression in colorectal cancer by activating nuclear factor $\kappa$ B and $\beta$-catenin. Oncogene 2005, 24:1021-1031 